\title{
LEAF MORPHOLOGY OF SOME SPECIES OF TRIBE PHASEOLEAE IN EGYPT
}

\author{
(Received: 9.12.2018)
}

\author{
By \\ Hanan S. Abd - El Maksoud \\ Flora and Phyto-taxonomy Researches Department, Horticultural Research Institute, Agricultural \\ Research Center, Giza, Egypt.
}

\begin{abstract}
Morphological variations of leaf characters of eight species of the tribe Phaseoleae belonging to three genera namely; Macroptilium, Phaseolus and Vigna were studied by macromorphological leaf characters and scanning electron microscopy (SEM) on both leaf surface. SEM clarified that there are seven sculptural patterns namely; unoulate, ruminate, reticulate, rugose, reticulate- rugulose, rugose tuberculate and sulcate - rugose. Based on leaf morphology and scanning electron microscopy on leaf surfaces, an artificial key was structured to distinguish the studied taxa.
\end{abstract}

Key words: leaf morphology, leaf surface, scanning electron microscopy (SEM), Tribe Phaseoleae, Macroptilium, Phaseolus, Vigna.

\section{INTRODUCTION}

The tribe Phaseoleae has the largest number of genera and the greatest economic importance in the family Fabaceae, a part from its genera being highly cultivated legumes and the others contain members that are of considerable importance as food, fodder and ornament (Bandyopadhyay et al., 2005).

The Phaseoleae belongs to the family Fabaceae, subfamily Faboideae (Leguminosae, Papilionoideae). Bentham (1865) divided the Papilionoideae into 11 tribes. Many workers now recognize more than the 11 tribes of Bentham (1865). Hutchinson (1964) lists 50 tribes by elevating many of Bentham's (1865) subtribes to tribes. Gillett et al. (1971) took up 17 tribes. Bentham's (1865) divided the Phaseoleae into six subtribes: Glycineae (includes genus Clitoria), Erythrineae, Galactieae, Diocleinae, Euphaseoleae (includes genus Phaseolus and Vigna and Cajaneae). The number of Phaseoleae genera has doubled since Bentham's time. Hutchinson's (1964) scheme differs slightly from Bentham's (1865), where he only added new genera and elevated most subtribes to the rank of tribe (where he added genus Macroptlium to tribe Phaseoleae). Grin taxonomy website (Angiosperm Phylogeny Group) recently divided subfamily Faboideae into 36 tribes and divided tribe Phaseoleae into 7 subtribes: Cajaninae, Clitoriinae, Diocleinae, Glycininae, Kennediinae, Ophrestiinae and
Phaseolinae; subtribe Clitoriinae includes genus Clitoria, while subtribe Phaseolinae included Macroptilium, Phaseolus and Vigna genera.

The Fabaceae are placed in the order Fabales according to most taxonomic systems, including the APG III system. (Angiosperm Phylogeny Group, 2009). The family now includes six subfamilies: Cercidoideae: 12 genera and $\sim 335$ species, Detarioideae: 84 genera and $\sim 760$ species, Duparquetioideae: 1 genus and 1 species. Dialioideae: 17 genera and $\sim 85$ species. Caesalpinioideae: 148 genera and 4400 species and Faboideae (Papilionoideae[5]): 503 genera and $\sim 14,000$ species. (The Legume Phylogeny Working Group (LPWG). 2017).

The tribe Phaseoleae is divided into 8 subtribes, one of these Phaseolinae includes 27 genera (three of which are Macroptilium, Phaseolus and Vigna). Many of these beans are cultivated for human and animal food, most importantly from the genera Phaseolus and Vigna. (Wojciechowski et al., 2004, and Delgado-Salinas et al., 2011).

Surface sculpturing by using Scanning Electron Microscope (SEM) technique may aid in solving problems of identity or relationship concerning taxa at various levels (Werker, 1997).

The currently accepted botanical name for the black-eyed peas Vigna unguiculata subsp. unguiculata, although was previously 
classified in the genus Phaseolus. Many varieties are usually distinguished by the different colors of their mature seeds ("USDA GRIN Taxonomy").

Although, yardlong bean resembles pole snap beans (Phaseolus vulgaris), it is botanically more closely related to southern cowpeas (Vigna unguiculata ). However, yardlong bean is much more a trailing and climbing plant than the southern pea, often reaching 9-12 feet in height (http://eol.org/pages/1231704/overview). The size and shape of the leaves varies greatly, making this an important feature for classifying and distinguishing cowpea varieties (Pottorff et al., 2012). The size and shape of juvenile leaves in particular are important taxonomic charaters in Eucalyptus (Pryor, 1976 ) with closely related species being differentiated by these traits (e.g Phillips and Reid 1980 ; Potts and Reid, 1985). Ostroumove (1990) studied the stomata types on leaves of some species that belong to the tribes Coriandreae and Scandiceae (Umbelliferae) in relation to taxonomy. Szujko-Lacza (1994) studied the leaf characters of Coriandrum sativum.

The possible evolutionary-ecological significance of surface sculpturing was briefly discussed. There is evidence that these features may be seen primarily under the aspects of reduced ability of plants to contaminate and as temperature control mechanisms of surfaces. SEM studies revealed an extraordinary diversity of epidermal plant surface structures. These characters, surprisingly, were little affected by the environmental conditions in which the plant grows and thus can be used systematically (Barthlott and Frolich, 1984).

Many micro-characters have diagnostic values only when characterizing lowest taxonomic categories (e.g. many types of cuticular striations, most of the frequent types of cell shapes, many types of widely distributed epicuticular crystalloids). There are many characters that could be used to characterize groups of related species, genera or taxonomic categories up to the sub-family levels. Some characters of the micro-morphology and orientation of epicuticular wax crystalloid are surprisingly high systematic significance. Wax platelets may have parallel orientation patterns resembling electromagnetic field lines around the stomata. This pattern, called Convallariatype (Cole and Behnke, 1975 and Barthlott, 1981). Dhalgren (1975), indicated the distribution of wax type (hatched) in the revised classification of angiosperms.

Bandyopadhyay et al. (2005) differed in Vigna unguiculata result, on the issue of macula-reticulate type of ornamentation. Leaf morphology on some species of family Apiaceae was studied by Abd El-Maksoud (2009).

Nath \& Dasgupta, (2015) recorded that SEM of spermoderm was found to be useful in establishing taxonomic and phylogenetic relationship in the Indian species of Vigna. The present investigation was an attempt to characterize seed coat on different species of tribe Phaseoleae in Egypt based on scanning electron microscopy (SEM).

\section{MATERIALS AND METHODS}

The current study was carried out during seasons 2016- 2017 on the leaves of eight species of Tribe Phaseoleae. Fresh leaves of the eight species were collected and identified by Flora and Phytotaxonomy Research Department, Horticultural Research Institute, Agricultural Research Center Giza, Egypt. These species were planted in the Medicinal and Aromatic Plants Research Department, Horticultural Research Institute, Agricultural Research Center, Dokki, Giza, Egypt. The detailed surface scan features were examined by using Scanning Electron Microscope (SEM) with different magnifications. Scanning was carried by JEOLJSM T 100 Model Scanning Electron Microscope, Central Laboratory, National Information and Documentation Center (NIDoC), Dokki, Giza, Egypt. Photos of the leaf were taken by a digital camera.

Measurements of external leaf characters were recorded separately for each species based on information mentioned by Abd El-Maksoud (2009), Barnard,(1969), Dhalgren (1975), Conway et al.(2001), Cook et al., (2005), Hutchinson (1964), Polhill and Raven (1981), Wojciechowski et al. (2004) and Pottorff et al. (2012).

\section{RESULTS AND DISCUSSION}

Morphological descriptions of the leaf of eight species of tribe Phaseoleae were studied and summarized in Table $(2 \& 3)$, plate 1 and plate 2, a \& b including Macromorphological features of leaf; color, shape, petiole (length ), stipules shape, stipules length $\mathrm{mm}$, base, leaflets shape, length, width, margin, apex, base, petiolules $\mathrm{mm}$, stipels $\mathrm{mm}$, texture of both surface and scanning electron microscopy (SEM) on leaf surface (upper and lower 
Table (1): The studied species of tribe Phaseoleae according to; The Plant List, BSBI List 2007and NRCS 2016.

\begin{tabular}{|c|c|c|c|}
\hline No. & Species & Synonyms & Common name \\
\hline 1 & $\begin{array}{l}\text { Macroptilium lathyroides (L.) } \\
\text { Urb. }\end{array}$ & Phaseolus lathyroides L. & $\begin{array}{c}\text { Phasey bean, bean - phasey, one-leaf clover, } \\
\text { wild bush-bean, phasemy bean, quail bean, } \\
\text { wild dolly bean }\end{array}$ \\
\hline 2 & Phaseolus acutifolius A. Gray & $\begin{array}{l}\text { Ph. acutifolius var. tenuifolius } \\
\text { A.Gray } \\
\text { Ph. tenuifolius (A. Gray) Wooton \& } \\
\text { Standl. }\end{array}$ & \begin{tabular}{|c|} 
Tepary bean, Fagiolo Tepary, Fasol \\
Ostrolistnaya, Fasola Ostrolistna, Frijol \\
Tepary, Kvasolya Zolotistaya, Papu, Tepari,
\end{tabular} \\
\hline 3 & Phaseolus lunatus L. & $\begin{array}{l}\text { Phaseolus bipunctatus Jacq } \\
\text { Ph. ilocanus Blanco } \\
\text { Ph. inamoenus L } \\
\text { Ph.limensis Macfad,Ph. Iunatus var. } \\
\text {.macrocarpus (Moench) Benth } \\
\text { Ph. macrocarpus Moench } \\
\text { Ph. portoricensis Spreng } \\
\text { Ph. puberulus Kunth } \\
\text { Ph. rosei Piper } \\
\text { Ph. saccharatus Macfad } \\
\text { Ph. tunkinensis Lour }\end{array}$ & $\begin{array}{c}\text { Lima bean, butter bean, sieva bean, } \\
\text { Madagascar bean }\end{array}$ \\
\hline 4 & Phaseolus vulgaris L. & $\begin{array}{l}\text { Phaseolus aborigineus Burkart } \\
\text { Ph. communis } \text { Pritz } \\
\text { Ph. compressus } \mathrm{DC} \\
\text { Ph. esculentus } \text { Salisb }\end{array}$ & Green bean, Kidney bean, Common bean \\
\hline 5 & Vigna luteola (Jacq.) Benth. & $\begin{array}{l}\text { Phaseolus luetolus (Jacq.) Gagnep } \\
\text { Ph. marinus Burm } \\
\text { Ph. maritimus Hassk } \\
\text { Vigna brachystachys Benth } \\
\text {.Vigna bukombensis Harms } \\
\text {.Vigna fischeri Harms } \\
\text { Vigna glabra Savi } \\
\text { Vigna helicopus (E. Mey.) Walp } \\
\text { Vigna jaegeri Harms } \\
\text { Vigna longepedunculata Taub } \\
\text { Vigna marina (Burm.) Merr } \\
\text { Vigna nigerica A. Chev } \\
\text { Vigna nilotica (Del.) Hook } \\
\text { Vigna oblonga Hook. f } \\
\text { Vigna repens Kuntze } \\
\text { Vigna villosa Savi }\end{array}$ & Dalrymple vigna, hairy cowpea \\
\hline 6 & Vigna radiata (L.) R. Wilczek & $\begin{array}{l}\text { Phaseolus abyssinicus Savi } \\
\text { Ph. aureus } \text { Roxb } \\
\text { Ph. chanetii (H.Lev.) H.Lev } \\
\text { Ph. hirtus } \text { Retz } \\
\text { Ph. novo-guineense Baker f } \\
\text { Ph. radiatus } \text { L } \\
\text { Ph. setulosus Dalzell } \\
\text { Ph. sublobatus } \text { Roxb } \\
\text { Vigna opistricha A.Rich } \\
\text { V. perrieriana R.Vig } \\
\text { V. sublobata } \text { (Roxb.) Bairig. \& al. }\end{array}$ & Mung bean, green gram, maash, moong \\
\hline 7 & Vigna unguiculata (L.) Walp. & & $\begin{array}{l}\text { Cowpea, black-eyed pea, southern pea, } \\
\text { yardlong bean, catjang }\end{array}$ \\
\hline 8 & $\begin{array}{l}\text { Vigna sesquipedalis (L.) } \\
\text { Frue. }\end{array}$ & $\begin{array}{l}\text { Vigna unguiculata (L.) Walp. subsp. } \\
\text { sesquipedalis (L.) Verdc }\end{array}$ & $\begin{array}{l}\text { Yard long bean, Asparagus bean, Chinese } \\
\text { long bean, snake bean }\end{array}$ \\
\hline
\end{tabular}

epidermis); type of stomata, stomatal leveling, type of trichomes and sculpture surface patterns.

Artificial key was structured based on macromorphological and micromorphological features of leaf surface to distinguish the studied species. 


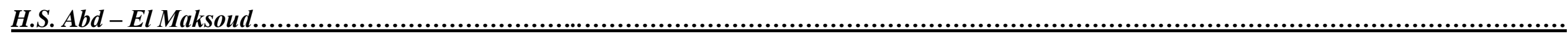

Table ( 2): Macromorphological description of the leaves of the studied species.

\begin{tabular}{|c|c|c|c|c|c|c|c|c|c|}
\hline \multicolumn{2}{|r|}{ Species Characters } & $\mathbf{1}$ & 2 & 3 & 4 & 5 & 6 & 7 & 8 \\
\hline \multirow{5}{*}{ ఏ్ } & Stipules shape & Lanceolate & Lanceolate & exstipulate & oblong & ovate-lanceolate & $\begin{array}{l}\text { peltate, ovate, or } \\
\text { obovate-oblong }\end{array}$ & exstipulate & exstipulate \\
\hline & $\begin{array}{l}\text { Stipules length } \\
\text { mm }\end{array}$ & $5-6$ & $2-3$ & - & $2-4$ & $3-8$ & $5-18$ & - & - \\
\hline & Arrangement & alternate & alternate & alternate & alternate & alternate & alternate & alternate & alternate \\
\hline & Petiole cm. & $2-5$ & $2-10$ & $6.5-9$ & $3.5-8.5$ & $1.5-6$ & $5-21$ & $2.5-10$ & $5-25$ \\
\hline & Base & Not swollen & swollen & Not swollen & swollen & negligible Swollen & Not swollen & Not swollen & amplexicau \\
\hline \multicolumn{2}{|c|}{ Type of lobed } & trifoliolate & trifoliolate & trifoliolate & simple or trifoliolate & trifoliolate & $\begin{array}{l}\text { trifoliolate } \\
\text { (sometimes 5foliolate) }\end{array}$ & trifoliolate & trifoliolate \\
\hline \multirow{9}{*}{ 苞 } & Shape & $\begin{array}{l}\text { Ovater to } \\
\text { lanceolate or } \\
\text { narrowly elliptic }\end{array}$ & $\begin{array}{l}\text { ovate to } \\
\text { ovate- } \\
\text { lanceolate }\end{array}$ & ovate & ovate & $\begin{array}{l}\text { ovate or ovate- } \\
\text { lanceolate }\end{array}$ & $\begin{array}{l}\text { elliptical, rhomboid or } \\
\text { ovate }\end{array}$ & ovate & ovate \\
\hline & Margin & entire & sinuate & entire & entire & entire & entire & entire & entire \\
\hline & Apex & $\begin{array}{l}\text { acute or } \\
\text { acuminate }\end{array}$ & acute & $\begin{array}{l}\text { acute or short- } \\
\text { acuminate }\end{array}$ & acute & acute or acuminate & acute or acuminate & acute & $\begin{array}{l}\text { acute or } \\
\text { acuminate }\end{array}$ \\
\hline & Base & $\begin{array}{l}\text { symmetrical } \\
\text { cuneate }\end{array}$ & subhastate & $\begin{array}{l}\text { cuneate or truncate } \\
\text {,terminal } \\
\text { asymmetric }\end{array}$ & \begin{tabular}{ll}
\multicolumn{3}{l}{ symmetrical,the } \\
lateral ones are \\
narrow \\
asymmetric.
\end{tabular} & $\begin{array}{l}\text { rounded or cuneate } \\
\text { symmetric }\end{array}$ & $\begin{array}{l}\text { broadly cuneate or } \\
\text { rounded, asymmetric }\end{array}$ & $\begin{array}{l}\text { terminal } \\
\text { symmetrical,centr } \\
\text { al asymmetrical }\end{array}$ & $\begin{array}{l}\text { acute or rounded } \\
\text { to obtuse }\end{array}$ \\
\hline & Petiolules mm & $1-3$ & $2-4$ & $3-5$ & $3-8$ & $2-3$ & $3-6$ & $2-5$ & $2-4$ \\
\hline & $\begin{array}{l}\text { Petiolules of } \\
\text { terminal } \mathbf{m m}\end{array}$ & $7-15$ & $8-15$ & $15-30$ & $14-30$ & $4-9$ & $15-28$ & $13-20$ & $3-6$ \\
\hline & Stipels $\mathbf{m m}$ & Linear 1-1.5 & $\begin{array}{l}\text { Linear up } \\
\text { to } 2\end{array}$ & Oblong 1-2 & Lanceolate 2-3 & Ovate 1 & conspicuous, $5-10$ & Lanceolate 2-3 & Ovate $2-3$ \\
\hline & Length cm & $3-8$ & $4-8$ & $3-19.5$ & $6-15$ & $2.5-11$ & $5-18$ & $3.4-6.5$ & $7-13.5$ \\
\hline & Width cm & $1-3.5$ & $2-5$ & $1-11$ & $3-11$ & $1-5$ & $3-15$ & $1.5-4$ & $4-9.5$ \\
\hline \multicolumn{2}{|c|}{ Color } & green & $\begin{array}{l}\text { slightly } \\
\text { green }\end{array}$ & $\begin{array}{l}\text { upper surface } \\
\text { dark green, dull, } \\
\text { lower surface pale } \\
\text { green or glaucous }\end{array}$ & $\begin{array}{l}\text { upper surface } \\
\text { green, lower } \\
\text { surface pale green }\end{array}$ & green & dark green & green & green \\
\hline \multirow{2}{*}{ 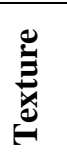 } & Upper surface & glabrous & glabrous & glabrous & pubescent & $\begin{array}{l}\text { sparsely } \\
\text { pubescent }\end{array}$ & glabrous or hairy & pubescent & nearly hairless \\
\hline & Lower surface & adpressed hairy & pubescent & glabrous & pubescent & $\begin{array}{l}\text { sparsely } \\
\text { pubescent }\end{array}$ & glabrous or hairy & pubescent & nearly hairless \\
\hline
\end{tabular}

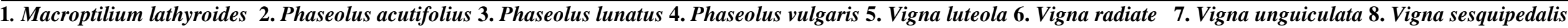


Table (3): Micromorphological description of the leaves of the studied species using Scanning Electron Microscopy (SEM).

\begin{tabular}{|c|c|c|c|c|c|c|c|c|c|}
\hline \multicolumn{2}{|c|}{$\begin{array}{r}\text { Species } \\
\text { Characters }\end{array}$} & 1 & 2 & 3 & 4 & 5 & 6 & 7 & 8 \\
\hline \multirow{5}{*}{ 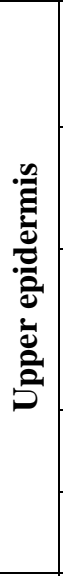 } & Type of stomata & anomocytic & anomocytic & anomocytic & anomocytic & paracytic & $\begin{array}{l}\text { paracytic and } \\
\text { actinocytic }\end{array}$ & actinocytic & actinocytic \\
\hline & $\begin{array}{l}\text { Stomatal } \\
\text { leveling }\end{array}$ & semiraised & $\begin{array}{l}\text { superficial \& } \\
\text { semi- } \\
\text { depressed }\end{array}$ & superficial & superficial & semiraised & $\begin{array}{l}\text { Superficial\& } \\
\text { semi-depressed }\end{array}$ & Superficial & superficial \\
\hline & $\begin{array}{l}\text { Type of } \\
\text { trichomes }\end{array}$ & $\begin{array}{l}\text { non-glandular } \\
\text { (vinca) }\end{array}$ & $\begin{array}{l}\text { non-glandular } \\
\text { (nux vimic) }\end{array}$ & $\begin{array}{l}\text { non-glandular } \\
\text { (nux vimic) }\end{array}$ & $\begin{array}{l}\text { non-glandular } \\
\text { (nux vimic) }\end{array}$ & $\begin{array}{l}\text { non-glandular ( } \\
\text { vinca )\& } \\
\text { glandular(digitali } \\
\text { s purpurea ) }\end{array}$ & $\begin{array}{l}\text { non-glandular } \\
\text { (nux vimic)\& } \\
\text { glandular(digitali } \\
\text { s purpurea ) }\end{array}$ & $\begin{array}{l}\text { non-glandular } \\
\text { (vinca) }\end{array}$ & glandular (mentha) \\
\hline & $\begin{array}{l}\text { Trichome } \\
\text { ornamentation }\end{array}$ & smooth & smooth & $\begin{array}{l}\text { warty \& } \\
\text { lignify }\end{array}$ & pusticulate & weak tuberculate & weak tuberculate & $\begin{array}{l}\text { warty \& } \\
\text { lignify }\end{array}$ & smooth \\
\hline & Sculpture & ruminate & $\begin{array}{l}\text { Reticulate- } \\
\text { rugulose }\end{array}$ & rugose & rugose & sulcate - rugose & rugose & rugulose & scrobiculate \\
\hline \multirow{5}{*}{ 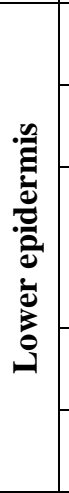 } & $\begin{array}{c}\text { Type of } \\
\text { stomata }\end{array}$ & anomocytic & anomocytic & anomocytic & anomocytic & paracytic & $\begin{array}{l}\text { paracytic and } \\
\text { actinocytic }\end{array}$ & actinocytic & $\begin{array}{l}\text { actinocytic \& } \\
\text { anomocytic }\end{array}$ \\
\hline & $\begin{array}{l}\text { Stomatal } \\
\text { leveling }\end{array}$ & $\begin{array}{l}\text { Superficial\& } \\
\text { semiraised }\end{array}$ & Depressed & $\begin{array}{l}\text { Superficial\& } \\
\text { semiraised }\end{array}$ & $\begin{array}{l}\text { raised \& } \\
\text { semiraised }\end{array}$ & semiraised & superficial & superficial & raised \&semiraised \\
\hline & $\begin{array}{l}\text { Type of } \\
\text { trichome }\end{array}$ & $\begin{array}{l}\text { Non- } \\
\text { glandular ( } \\
\text { senna ) }\end{array}$ & $\begin{array}{l}\text { non-glandular } \\
\text { (vinca) }\end{array}$ & $\begin{array}{l}\text { non-glandular } \\
\text { (nux vimic) }\end{array}$ & $\begin{array}{l}\text { non-glandular } \\
\text { (nux vimic) }\end{array}$ & $\begin{array}{l}\text { non-glandular ( } \\
\text { vinca }) \& \\
\text { glandular(digitali } \\
\text { s purpurea ), }\end{array}$ & $\begin{array}{l}\text { non-glandular } \\
\text { (nux vimic)\& } \\
\text { glandular(digitali } \\
\text { s purpurea }\end{array}$ & $\begin{array}{l}\text { non-glandular } \\
\text { (vinca) }\end{array}$ & glandular (mentha) \\
\hline & $\begin{array}{l}\text { Trichome } \\
\text { ornamentation }\end{array}$ & Smooth & Smooth & Smooth & Smooth & weak tuberculate & weak tuberculate & lignify & Smooth \\
\hline & Sculpture & $\begin{array}{l}\text { weak } \\
\text { ruminate }\end{array}$ & Reticulate & ruminate & $\begin{array}{l}\text { rugose - } \\
\text { tuberculate }\end{array}$ & sulcate - rugose & unoulate & scalariform & scrobiculate \\
\hline
\end{tabular}

1. Macroptilium lathyroides 2. Phaseolus acutifolius 3. Phaseolus lunatus 4. Phaseolus vulgaris 5. Vigna luteola 6. Vigna radiate 7. Vigna unguiculata 8. Vigna sesquipedalis 


\section{Macroptilium lathyroides (L.) Urb.}

Leaves trifoliolate; leaflets mostly entire margin, ovate to lanceolate, or narrowly elliptic, 3-8 cm long; 1-3.5 cm wide, upper surface glabrous, lower surface adressed hairy, petioles $1-5 \mathrm{~cm}$ long, stipules lanceolate, 5-6 $\mathrm{mm}$. long, lateral leaflets sometimes slightly lobed towards the base. Upper epidermis has stomatal type; anomocytic with superficial semi-depressed level and with ruminate sculpture pattern, trichomes; non-glandular (vinca), smooth ornamentation. Lower epidermis has anomocytic stomatal type with superficial and semiraised level and with weak ruminate sculpture pattern, trichomes; non-glandular (senna), smooth ornamentation.

\section{Phaseolus acutifolius A. Gray}

Leaves alternate, trifoliolate; tapering, pointtipped stipules and are green, sparsely hairy, stipules lanceolate, $2-3 \mathrm{~mm}$ long, appressed to stem; petiole $2-10 \mathrm{~cm}$ long; stipels linear, up to $2 \mathrm{~mm}$ long; leaflets ovate to ovate-lanceolate, 4$8 \mathrm{~cm} \times 2-5 \mathrm{~cm}$, acute, sinuate margin, usually pubescent below, lateral ones sometimes slightly subhastate on one side, giving them a lopsided appearance, attenuate at apex. Upper epidermis has stomatal type; anomocytic with superficial and semi-depressed level and with reticulaterugulose sculpture pattern, trichomes; nonglandular (nux vimic), smooth ornamentation. Lower epidermis has anomocytic stomatal type with depressed level and with reticulate sculpture pattern, trichomes; non-glandular (vinca), smooth ornamentation.

\section{Phaseolus lunatus $\mathrm{L}$.}

The leaves alternate, exstipulate, trifoliate with ovate leaflets, $3-19.5 \mathrm{~cm}$ long x 1-11 cm wide, the apex acute or short-acuminate, the margins entire; upper surface dark green, dull, glabrous, with slightly prominent venation; lower surface pale green or glaucous, glabrous, with the primary and secondary venation prominent; terminal leaflet $5.5-11 \times 3.5-7.5 \mathrm{~cm}$, rhombic or deltate, the base cuneate or truncate; lateral leaflets asymmetrically deltate, the base truncate; petiolules 3-5 $\mathrm{mm}$ long, pubescent; petioles $6.5-9 \mathrm{~cm}$ long, sulcate, puberulent, the base slightly broadened. Upper epidermis has stomatal type; anomocytic with superficial level, with rugose sculpture pattern, trichomes; non-glandular (nux vimic), warty and lignify ornamentation. Lower epidermis has anomocytic stomatal type with superficial and semiraised level and with ruminate sculpture pattern, trichomes; non-glandular (nuxvimic), smooth

\section{ornamentation.}

\section{Phaseolus vulgaris $\mathrm{L}$.}

Leaves alternate, upper surface green, lower surface pale green. The leaf may be simple (have only one blade per petiole) or usually trifoliate which are trifoliate with ovate leaflets, smoothedged leaflets, each 6-15 cm long and 3-11 cm wide. acute apex, wider and symmetrical, while the lateral ones are narrow and asymmetric., margin entire. There may be two simple leaves or one compound leaf attached at a spot on the stem called a node. Where the stem and leaf join, there is a swollen area of the petiole (pulvinus). At night the bean leaves fold together and down toward the soil; at dawn the leaves unfold and lifted into the sun. These leaves are fleshy, pubescent on both surfaces. Upper epidermis has stomatal type; anomocytic with superficial level and with rugose sculpture pattern, trichomes; non-glandular (nux vimic), pusticulate ornamentation. Lower epidermis has anomocytic stomatal type with raised and semiraised level and with rugose - tuberculate sculpture pattern, trichomes; non-glandular (nux vimic), smooth ornamentation.

\section{Vigna luteola (Jacq.) Benth.}

Leaves trifoliolate, with leaflets ovate or ovate-lanceolate, $2.5-11 \mathrm{~cm}$ long, $1-5 \mathrm{~cm}$ wide, acute or acuminate at the apex, rounded or cuneate at the base, sparsely pubescent on both surfaces, petiole $2-8 \mathrm{~cm}$ long; rachis $0.5-1.2 \mathrm{~cm}$ long; petiolules 2-3 mm long; stipules ovatelanceolate, 3-8mm long, $2 \mathrm{~mm}$ wide, shortly bilobed at the base. Both surfaces of epidermis are the same micromorphological features of leaf. Upper epidermis has stomatal type; paracytic with semiraised level and with sulcate - rugose sculpture pattern, trichomes; nonglandular (vinca) and glandular (digitalis purpurea), weak tuberculate ornamentation. Lower epidermis has paracytic stomatal type with semiraised level and with sulcate - rugose sculpture pattern, trichomes; non-glandular (vinca) and glandular (digitalis purpurea), weak tuberculate ornamentation.

\section{Vigna radiata (L.) R. Wilczek}

Slightly pubescent herb. Leaves alternate, trifoliolate (sometimes 5 foliolate), dark green; stipules 5-18 $\mathrm{mm} \times 3-10 \mathrm{~mm}$, peltate, ovate, or obovate-oblong; petiole 5-21 cm long, rachis $1.5-4.5 \mathrm{~cm}$ long; stipels conspicuous, $5-10 \mathrm{~mm}$ long; petiolules 3-6 mm long; leaflets entire, 5$18 \mathrm{~cm} \times 3-15 \mathrm{~cm}$, elliptical, rhomboid or ovate, base broadly cuneate or rounded, apex acuminate, glabrous or hairy on both surfaces, 


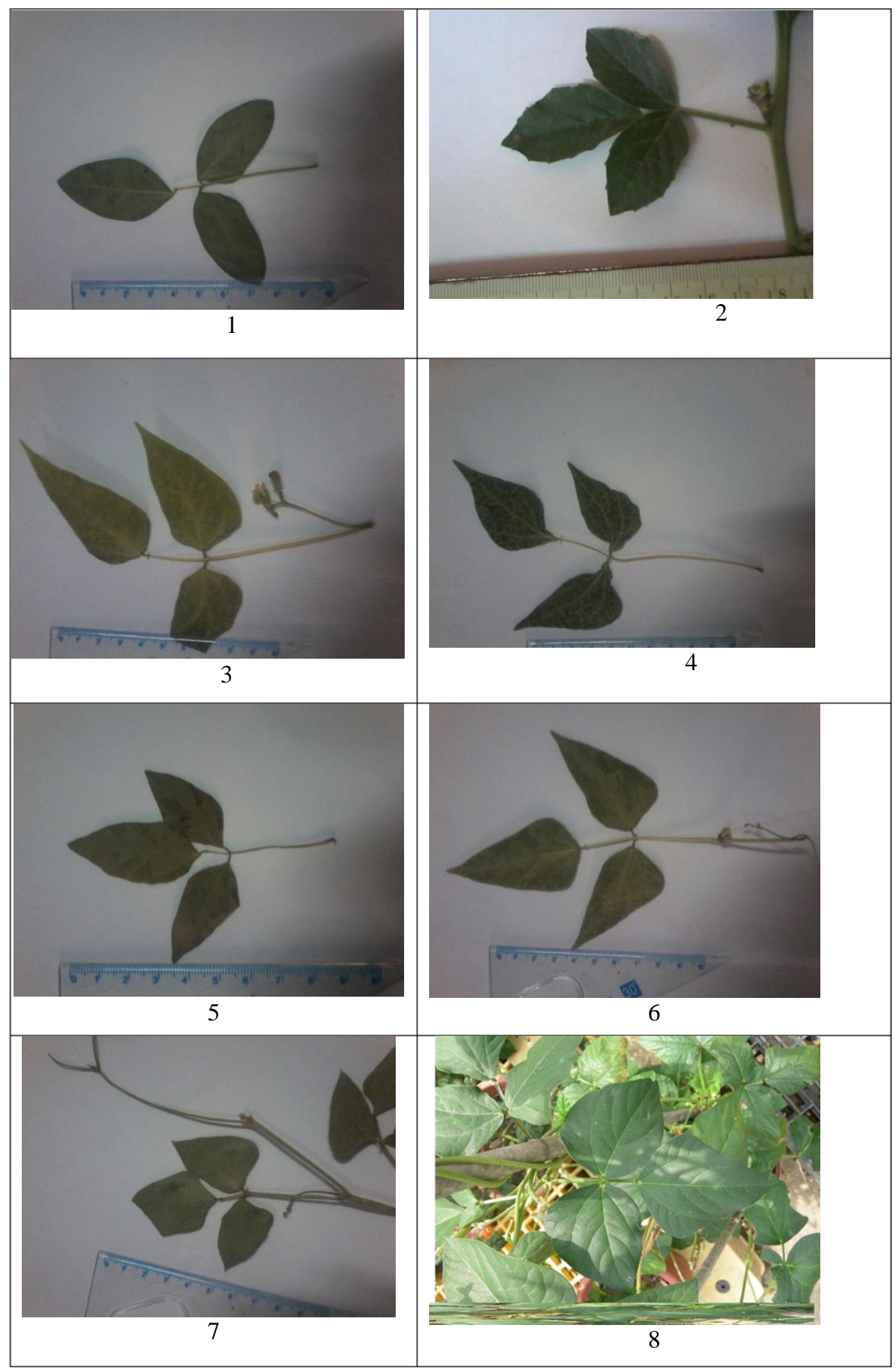

Plate (1): Shapes of leaf; 1. Macroptilium lathyroides 2.Phaseolus acutifolius 3. Phaseolus Iunatus 4. Phaseolus vulgaris 5. Vigna luteola 6. Vigna radiata 7. Vigna unguiculata 8. Vigna sesquipedalis 


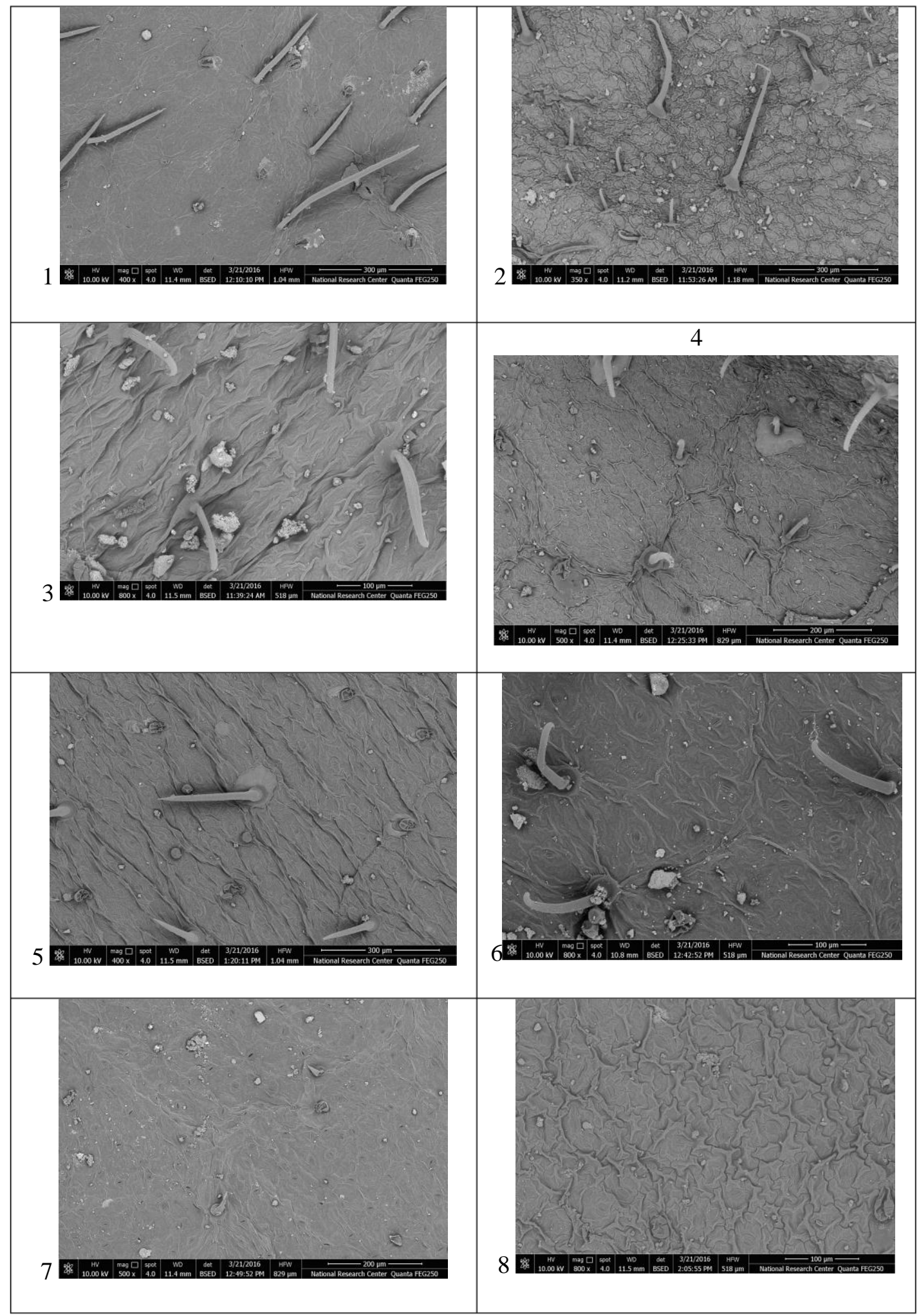

Plate. (2a): SEM on leaf epidermis; upper surface. 1.Macroptilium lathyroides 2. Phaseolus acutifolius 3. Phaseolus lunatus 4. Phaseolus vulgaris 5. Vigna luteola 6. Vigna radiata 7. Vigna unguiculata 8. Vigna sesquipedalis 


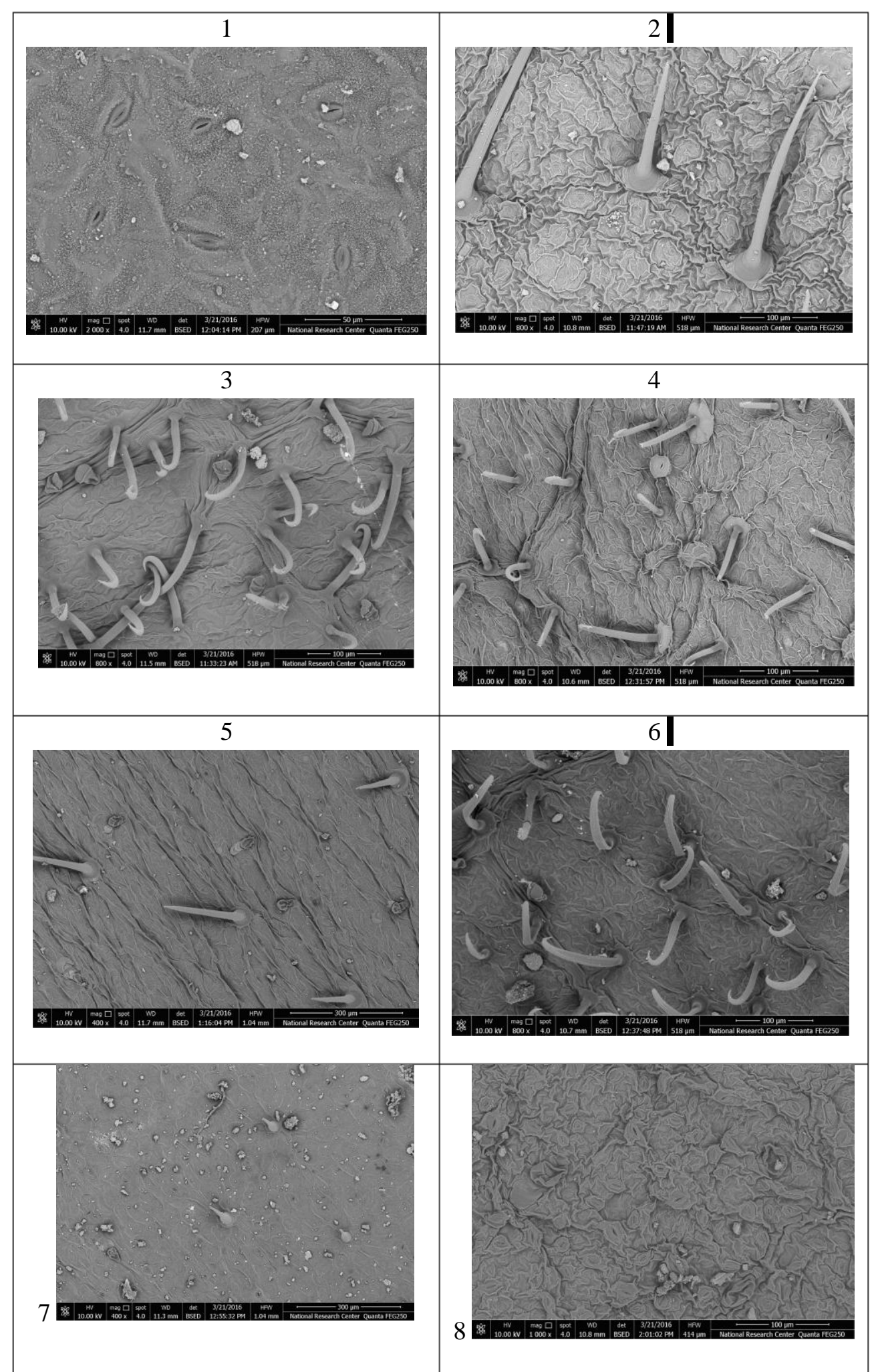

Plate. (2b): SEM on leaf epidermis; lower surface 1. Macroptilium lathyroides 2.Phaseolus acutifolius 3. Phaseolus lunatus 4. Phaseolus vulgaris 5. Vigna luteola 6. Vigna radiata 7. Vigna unguiculata 8. Vigna sesquipedalis 
distinctly 3-veined from the base, the lateral leaflets unequal-sided. Upper epidermis has stomatal type; paracytic and actinocytic with superficial and semi-depressed level and with rugose sculpture pattern, trichomes; nonglandular (nux vimic) with weak tuberculate ornamentation and glandular (digitalis purpurea). Lower epidermis has paracytic and actinocytic stomatal type with superficial level and with unoulate sculpture pattern, trichomes; nonglandular (nux vimic) with weak tuberculate ornamentation and glandular (digitalis purpurea).

\section{Vigna unguiculata (L.) Walp}

There is a large morphological diversity found within the crop, and the growth conditions and grower preferences for each variety vary from region to region (Singh et al., 1997). Variability in plant morphology of the different accessions is high. There are three types according to their uses: for grain, forage or dual-purpose.

Leaves alternate, exstipulate, trfioliolate with petioles $5-25 \mathrm{~cm}$ long. The lateral leaflets are opposite and asymmetrical, while the central leaflet is symmetrical and ovate. 1.5-4 $\mathrm{cm}$. x 3.4-6.5 cm, petiolule $2-5 \mathrm{~mm}$. Terminal leaflet $6.32-7.52 \mathrm{~cm}$. width , $8.72-10.40 \mathrm{~cm}$ length, petiolule length $1.3-2 \mathrm{~cm}$. Upper epidermis has stomatal type; actinocytic with superficial level and with rugulose sculpture pattern, trichomes; non-glandular (vinca), warty \& lignify ornamentation. Lower epidermis has actinocytic stomatal type with superficial level and with scalariform sculpture pattern, trichomes; non-glandular (vinca), lignify ornamentation.

\section{Vigna sesquipedalis (L.)Frue.}

Leaves green, exstipulate, alternately arranged along the stem and are trifoliate, the first two leaflets being asymmetrical in shape with the terminal leaflet being ovate and symmetrical in shape, nearly hairless leaflets , 7$13.5 \mathrm{~cm}$ long, 4-9.5 $\mathrm{cm}$ wide, margin entire, base acute or rounded to obtuse, apex acute or acuminate, petiole $5-25 \mathrm{~cm}$ long. Upper epidermis has stomatal type; actinocytic with superficial level and with scrobiculate sculpture pattern, trichomes; glandular (mentha), smooth ornamentation. Lower epidermis has anomocytic and actinocytic stomatal type with raised and semiraised level and with scrobiculate sculpture pattern, trichomes; glandular (mentha), smooth ornamentation.

Surface sculpturing by using Scanning Electron Microscope (SEM) technique may aid in solving problems of identity or relationship concerning taxa at various levels (Werker, 1997).

Artificial key was structured based on macromorphological and micromorphological features of leaf surface to distinguish the studied species.

A. Leaf exstipulate, leaflet shape ovate

B. Stipels shape ovate with upper epidermis; stomata actinocytic and lower epidermis; stomata actinocytic \& anomocytic. Both surface; sculpture scrobiculate and trichomes glandular (mentha) ...... Vigna sesquipedalis

BB. Stipels shape oblong with upper epidermis; sculpture rugose and lower epidermis; sculpture ruminate. Both surface; stomata anomocytic and trichomes non- glandular (nux vimic) .......... Phaseolus lunatus

BBB. Stipels shape Lanceolate with upper epidermis; sculpture rugulose and lower epidermis; Sculpture scalariform. Both surface; stomata actinocytic and trichomes non-glandular (vinca)............Vigna unguiculata

AA. Leaf stipulate

C. Leaf dark green with upper epidermis; sculpture rugose and lower epidermis ; sculpture unoulate. Both surface; stomata paracytic and actinocytic and trichomes non-glandular (nux vimic) \& glandular (digitalis purpurea)..........Vigna radiate

CC. Leaf upper surface green with sculpture rugose, lower surface pale green with sculpture rugose - tuberculate. Both surface; stomata anomocytic and trichomes non-glandular (nux vimic) ....... Ph. Vulgaris

CCC. Leaf green

D. Leaflet margin sinuate with upper surface sculpture reticulate- rugulose, lower surface sculpture reticulate. Both surface; stomata anomocytic and trichomes non-glandular .......... Ph. acutifolius

DD. Leaflet margin entire

E. Both surface; sculpture sulcate - rugose, stomata paracytic and trichomes nonglandular (vinca) \& glandular (digitalis purpurea) ......... Vigna luteola

EE. Both surface; sculpture ruminate, stomata anomocytic and trichomes nonglandular ......Macroptilium lathyroides

In conclusion, Macromorphology and micromorphology of leaf are found characteristic to each species and appeared to be species- 
specific. So SEM study of leaf could be useful in establishing taxonomic and phylogenetic relationship among the studied species.

\section{Acknowledgement}

I would like to express my thanks to Dr. Sherif S. G. El- Khanagry, Emeritus Professor, Flora and Phytotaxonomy Research Department, Horticultural Research Institute, Agricultural Research Center, for his help and supplying the plants under study.

\section{REFERENCES}

Abd El-Maksoud H. S. (2009). Taxonomic studies on some species of Apiaceae (Umbelliferae). (Ph. D.) Thesis p. 182. Department of Agricultural Botany, Faculty of Agriculture, Cairo University, Egypt.

Angiosperm Phylogeny Group (2009). "An update of the angiosperm phylogeny group classification for the orders and families of flowering plants: APG III" (PDF). Botanical J. Linnean So., 161 (2): 105121.doi:10.1111/j.1095-339.2009.00996.x. Retrieved on February 42014. Bandyopadhyay B.; Santra, S. C. and Kato M. (2005). Legume Res., 28 (4): 235-243

Bandyopadhyay B., Santra S. C. and Kato M. (2005). Legume Research. 28(4):235-243.

Barnard C. (1969). Herbage plant species. Aust. Herbage Plant Registration Authority; Canberra, CSIRO Aust., Divn. of Plant Ind.

Barthlott W. (1981). Epidermal and seed surface characters of plants: Systematic applicability and some evolutionary aspects. Nordic J. Bot., Copenhagen (1): 345-355

Barthlott W. and Frolich J. (1984). Microstructural features of seed surfaces (ed. V. H. Heywood \& Moore, D. M.), Academic Press, London. pp. 95-105.

Bentham F. (1865a). Leguminosae. Pages 434600 In: JG. Bentham and J. D. Hooker. (eds) Genera Plantarum. Vol. 1, Part 2. Reeve \& Co., London, pp. 434-600.

Conway M. J., Mc Cosker K., Osten V., Coaker S. and Pengelly B. C. (2001). Butterfly Pea - A Legume Success Story in Cropping Lands of Central Queensland. In; Rowe B., Mendham N. and Donaghy D. (eds). Proceedings of the 10th Australian Agronomy Conference,. Hobart, Tasmania. (Eds B Rowe, D Donaghy, $\mathrm{N}$ Mendham) (Australian
Society of Agronomy/Regional Institute Ltd: Gosford, NSW)

"BSBI List 2007". Botanical Society of Britain and Ireland. Archived from the original (xls) on 2015-01-25. Retrieved 2014-1017.

Cook B. G., Pengelly B. C., Brown S. D., Donnelly J. L., Eagles D. A., Franco M. A., Hanson J., Mullen B. F., Partridge I. J., Peters M., and Schultze-Kraft R. (2005). Tropical forages. CSIRO, DPI\& F (Qld), CIAT and ILRI, Brisbane, Australia

Cole G. T. and Benhnke H. D. (1975). Electron microscopy and plant systematics. Taxon., 24: 315

Dhalgren R. (1975). A system of classification of the Angiosperms to be used to demonstrate the distribution of characters. Botaniska Notiser, 128: $119-147$

Delgado-Salinas A., Thulin M., Pasquet R., Weeden N. and Lavin M. (2011). Vigna (Leguminosae) sensu lato: the names and identities of the American segregate genera. Am. J. Bot., 98 (10): 1694-715.

Gillett J. B., Polhill R. M. and Verdcourt. B. (1971). Leguminosae (Part 3) subfamily Papilionoideae (l) In: E. Milne-Redhead and R. M. Polhill, (eds). Flora of Tropical East Africa. Whitefriars Press, London and Tonbridge,UK.

Grin taxonomy website (Angiosperm Phylogeny Group):

https://npgsweb.arsgrin.gov/gringlobal/tax onomyfamily .aspx ?type $=$ tribe $\&$ id $=1507$

Hutchinson J. (1964). The genera of flowering plants. Dicotylédones, Vol. 1. Oxford University Press, London.

Nath D. and Dasgupta T. (2015). Study on seed coat of some Vigna species following scanning electron microscopy (SEM). Int'1 J. Sci. Res. Publi., 5, (9): 2250-3153.

Natural Resources Conservation Service (NRCS). (2016). www.nrcs.usda.gov

Ostroumove T.A. (1990). Stomatal types in the Umbelliferae in relation to taxonomy of tribe Conianreae \& Scandiceae. Fedds Report 101 (7-8): 409-416.

Polhill R.M. and Raven P.H. (1981). Advances in Legume Systematics. Royal Botanic Gardens, Kew, UK.

Phillips R. L. and Reid J. B. (1980). Clinal variation btween Eucalyptus viminalis Labill. and Eucalyptus dalrympleana Maiden. Aust. J. Bot., 28: 329-342.

Potts B. M. and Reid J. B. (1965b). Variation in 
the Eucalyptus gnii-archerii complex. 11 The origin of variation. Aust. J. Bot., 33: 519-541.

Pottorff M. E., Jeffrey D., Fatokun, Ch., Roberts P. A. and Close T. J. (2012). "Leaf morphology in Cowpea [Vigna unguiculata (L.) Walp]: QTL analysis, physical mapping and identifying a candidate gene using synteny with model legume species". BMC Genomics, 13: 234.

Pryor L. D. (1976). The biology of Eucalyptus. Edward Arnold, London, U. K., pp82. Rowe B., Mendham N. and Donaghy D. (eds). Proceedings of the $10^{\text {th }}$ Australian Agronomy Conference, Hobar,

Singh B. B., Chambliss O. L. and Sharma B. (1997). "Recent advances in cowpea breeding". In Singh B. B., Mohan D. R., Dashiell K. E., Jackai L. E. N. Advances in Cowpea Research (PDF). Ibadan, Nigeria: International Institute of Tropical Agriculture and Japan International Research Center for Agricultural Sciences.
Szujko-Lacza J. (1994). Architecture and inner structure of the cariandram sativum L., Akad mial Kiado. Budapest, Prielle Korne liau. pp. 19-35.

The Legume Phylogeny Working Group (LPWG) (2017). "A new subfamily classification of the Leguminosae based on a taxonomically comprehensive phylogeny". Taxon., 66 (1):44-77. doi:10.12705/661.3.

The Plant List". a working list of all known plant species. Version 1, released in December 2010, http://www.theplantlist.org/1/

USDA GRIN Taxonomy for Plants database: https://www.usgbc.org/resources/usdagrin-taxonomy-plants-database

Werker E. (1997). Seed Anatomy. Gebrvder Borntraeger, Berlin, Germany. 359 p.

Wojciechowski M.F., Lavin M., Sanderson M. J. (2004). "A phylogeny of legumes (Leguminosae) based on analysis of the plastid matK gene resolves many wellsupported subclades within the family". Am. J. Bot., 91 (11):1846-1862.

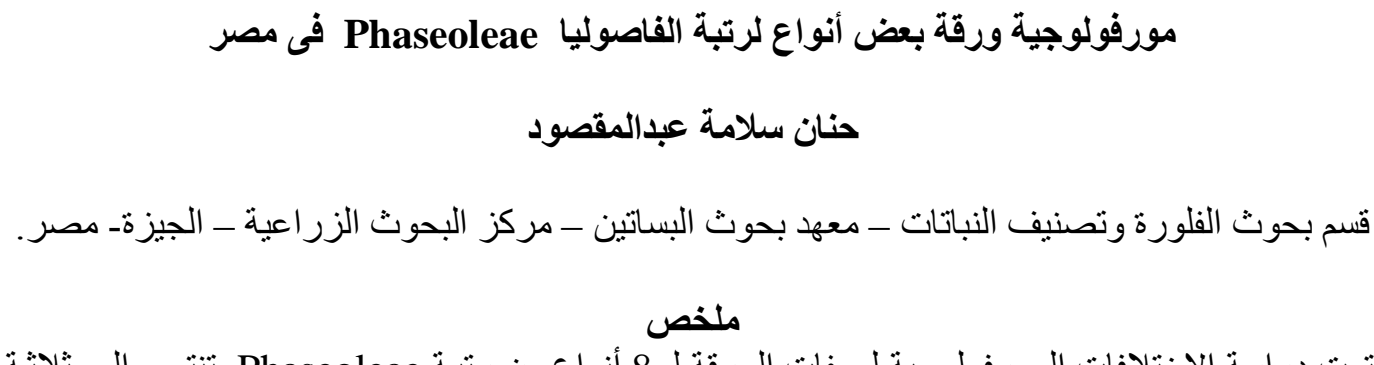

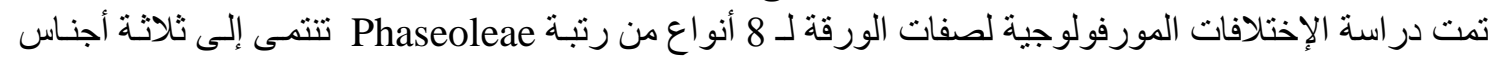

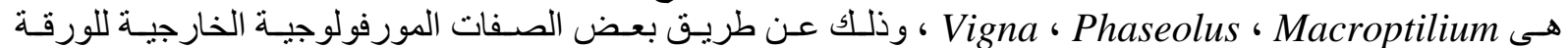

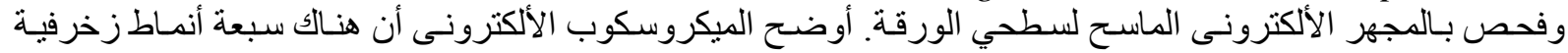

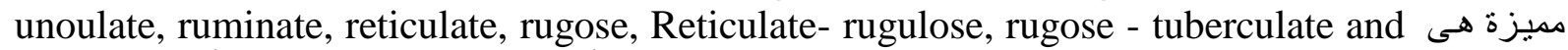
sulcate - rugose

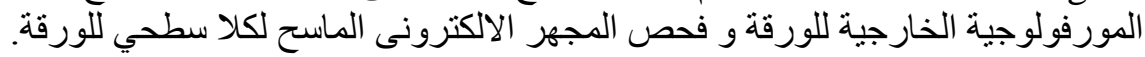
المجلة العلمية لكلية الزراعة - جامعة القاهرة - المجلد (69) العدد الرابع (أكتوبر 2018): 341-352. 